doi:10.1017/\$1041610219000796

\section{Person-centered attitudes mediate the association between dementia knowledge and help-giving intentions in Singapore}

An awareness-raising approach to building dementia-friendly community assumes that provision of accurate and complete information about dementia contributes to improved dementia literacy and reduced stigma among the public, which in turn encourages help-giving behavior toward people with dementia (Rees, 2015). While this assumption is consistent with the Theory of Planned Behavior (Ajzen, 1991), it has not been tested empirically.

We investigated whether knowledge of symptoms of dementia exerts an effect on help-giving intentions through person-centered or stigma attitudes, using data from a pilot study sample of 109 people aged 21 years or older who were identified as citizens or permanent residents of Singapore. They were intercepted in public spaces in three study communities and voluntarily responded to a survey. Ethical approval was obtained from Singapore University of Technology and Design's Institutional Review Board.

Help-giving intention was assessed with the scenario: "Imagine you are walking through your neighborhood and you notice a person in their 70s who you suspect is lost because they seem confused and agitated. How likely, or unlikely, is it that you would approach the person to offer help?" Responses were dichotomized into unlikely (0) versus likely $(1 ; n=89,81.7 \%)$. Symptom knowledge was assessed as the number of correctly identified symptoms from a list of eight real and two dummy symptoms $(\mathrm{M}=4.39, \mathrm{SD}=2.29) \quad(\mathrm{NatCen}$ Social Research, 2015). Stigma attitudes were assessed by three items rated from strongly agree (5) to strongly disagree (1): (a) I would avoid contact with someone if I thought they had dementia; (b) if I had just been told I had the first signs of dementia, I would feel ashamed; and (c) people diagnosed with dementia could have done something to avoid their condition (Phillipson et al., 2014). Item scores were summed ( $M=7.95$, $\mathrm{SD}=2.20 ; \alpha=0.57)$. Person-centered attitudes was assessed with two statements rated from strongly agree (5) to strongly disagree (1): (a) people with dementia have care and concern for other people, and (b) people with dementia are respected for their wisdom from years of life experience (Phillipson et al., 2014). Item scores were summed $(M=6.85, \mathrm{SD}=1.58 ; \alpha=0.58)$.
The direct association of symptom knowledge to help-giving intention and the indirect association via attitudes were estimated using a path model approach. Parallel multiple mediator analyses were performed in SPSS and PROCESS v.3.3, using maximum likelihood logistic regression with bootstrapping 5,000 samples and bias-corrected method to obtain $95 \%$ confidence intervals for the indirect effect, expressed in a log-odds metric (Hayes, 2018).

Of the 109 respondents, $62 \%(n=68)$ were aged between 21 to 54 years, $56 \%(n=61)$ were female, $69 \%(n=75)$ were Chinese, $60 \%(n=65)$ were residing in a Housing and Development Board flat of four or less rooms, and $52 \%(n=57)$ had at least a postsecondary education. Approximately $49 \% \quad(\mathrm{n}=53)$ personally know someone with dementia and $28 \%(n=31)$ care for a relative with dementia.

Greater symptom knowledge was associated with greater person-centered $(\mathrm{B}=0.21, \mathrm{SE}=0.07,95 \%$ CI $[0.07,0.35])$ but not stigma $(B=-0.02$, $\mathrm{SE}=0.10,95 \%$ CI $[-0.21,0.17])$ attitudes. Greater person-centered attitudes $(\mathrm{B}=0.66$, $\mathrm{SE}=0.22,95 \% \mathrm{CI}[0.22,1.10])$ were significantly associated with likely help-giving intention, but stigma attitudes $(\mathrm{B}=-0.22, \mathrm{SE}=0.16,95 \%$ CI $[-0.54,0.10])$ were not. Greater knowledge was indirectly associated with likely help-giving intention through more person-centered attitudes $(B=0.14, S E=3.14,95 \% \mathrm{CI}[0.03,0.60])$. Indirect pathway from knowledge to help-giving intention through stigma attitudes was nonsignificant $(\mathrm{B}=0.005, \mathrm{SE}=0.55,95 \%$ CI $[-0.10,0.10])$. The direct effect of greater knowledge on likely help-giving intention was significant $(B=0.43$, $\mathrm{SE}=0.17,95 \%$ CI $[0.09,0.77])$.

This letter offers preliminary support for the underlying assumption of the awareness-raising approach to building dementia-friendly community by presenting evidence that greater knowledge of dementia symptoms was associated with a greater likelihood that members of the public would give help to a person with dementia, through its association with person-centered attitudes toward dementia. Person-centered attitudes may represent a plausible factor instrumental in the formation of helping intentions and possibly motivating actual helping behavior. When the public has an opportunity to observe people with dementia fulfilling multiple, personal, familial, and social roles, they may better see the person behind dementia symptomology and be induced to act prosocially 
toward that person. To better inform awarenessraising efforts, we recommend that future studies replicate and extend this finding with large, population-based samples and longitudinal data to determine the temporal associations.

\section{Conflict of interest}

None.

\section{Description of authors' roles}

A. Lane designed the study, supervised the data collection, conceptualized the paper idea, supervised the statistical analysis, and wrote the paper. F. Yu was responsible for data collection and carrying out the statistical analysis. She assisted with conceptualizing the paper idea and writing the article.

\section{Acknowledgments}

This pilot study was supported by the Lee Li Ming Programme in Ageing Urbanism, Lee Kuan Yew Centre for Innovative Cities, Singapore University of Technology and Design. We thank and express appreciation to Dr Belinda Yuen, Professorial Fellow and Head of the Lee Li Ming Programme in Ageing Urbanism, who helped to design the pilot study, and also to Ms Katherine Loh, who provided assistance with study design and data collection.

\section{References}

Ajzen, I. (1991). The theory of planned behavior. Organizational Behavior and Human Decision Processes, 50, 179-211. doi: 10.1016/0749-5978(91)90020-T.

Hayes, A. F. (2018). Introduction to Mediation, Moderation, and Conditional Process Analysis: A Regression-based Perspective (2nd ed.). New York: The Guilford Press.

NatCen Social Research. (2015). Attitudes to dementia findings from the 2015 British Social Attitudes survey. Public Health England. Available at http://www.bsa .natcen.ac.uk/media/39130/bsa-33-attitudes-to-dementia .pdf; last accessed 15 May 2019.

Phillipson, L., Magee, C. A., Jones, S. C. and Skladzien, E. (2014). Correlates of dementia attitudes in a sample of middleaged Australian adults. Australasian fournal on Ageing, 33, 158-163. doi: 10.1111/j.1741-6612.2012.00624.x.

Rees, G. (2015). Dementia friendly communities: global initiatives and future directions. Paper presented at 25th Anniversary Celebration Alzheimer's Disease Association of Singapore. Available at https://www.dementiaallianceinternational.org/dementia-friendly-communities-globalinitiatives-and-future-directions-by-glenn-rees-am/; last accessed 15 May 2019

Anna P. Lane and Freya C.H. Yu

Lee Kuan Yew Centre for Innovative Cities, Singapore University of Technology and Design, Singapore

Correspondence should be addressed to: Anna P. Lane, Lee Kuan Yew Centre for Innovative Cities, Singapore University of Technology and Design, 8 Somapah Road, 487372, Singapore. Phone: +65 64348205.

Email: anna_lane@sutd.edu.sg. 